\title{
Research on Location Routing Optimization of Rescue Center After Earthquake Based on Two-stage Heuristic Algorithm
}

\author{
Siyun Zeng ${ }^{1, *}$, Chong Ye ${ }^{2}$ \\ ${ }^{1,2}$ School of Economics and Management, Fuzhou University \\ *Corresponding author. Email:540142140@qq.com
}

\begin{abstract}
When a large-scale earthquake occurs, due to road damage and limited resources, the injured often cannot get timely treatment. At this time, local governments at all levels need to quickly carry out rescue work, immediately activate emergency plans, and establish material distribution points and rescue centers.Reasonable location of emergency facilities can reduce the distance between evacuees and resources. This paper establishes a problem model related to rescue center location and rescue route. First, minimize the cost of rescue center location as the first stage optimization goal, and use immune optimization algorithm to solve it, and then select a reasonable rescue center location. Sceondly, a two-stage mathematical programming model for post-earthquake wounded rescue with the shortest total rescue routes is established, simulated annealing algorithm and ant colony algorithm are designed to solve the problem, and the results of these two algorithms are compared and analyzed.
\end{abstract}

Keywords: Wounded rescue, Rescue center location, Rescue route optimization, Immune optimization algorithm

\section{INTRODUCTION}

Earthquakes and other natural disasters occur without warning, but they often have a huge impact on society and seriously threaten people's lives and property safety. Scientific site selection scheme and effective scheduling of rescue vehicles for the wounded can not only improve the rescue efficiency after the earthquake, reduce casualties and emergency costs, but also realize efficient and intensive use of rescue resources. Therefore, it is one of the core research contents of earthquake rescue planning to select a reasonable and scientific rescue center location and optimize the path according to the rescue situation of the wounded after the earthquake, which has important practical significance.

The location of emergency facilities such as rescue centers is a type of complex decision-making problem. Scholars will consider multiple target decisions into the emergency facility location model. . Jomon A. P. and L. MacDonald (2016) established a stochastic modeling framework to determine the location of the distribution center of an emergency warehouse, and to improve the rescue and disaster relief capabilities in the case of disasters with little or no warning[7]. Huiling Zhong et al. (2013) used a greedy algorithm to solve the $\alpha$ -robust arc coverage model, comprehensively considering the number of facilities and the weighted coverage ratio, and selected a reasonable emergency facility location from the emergency facility candidate addresses to quickly respond to dangerous goods accident[6]. Firat, K. et al. (2015) establish a mixed integer The linear programming model makes decisions on the location of the evacuation site, so that people can quickly evacuate to nearby facilities in a short time[3]; Huali Sun et al. (2020) considered the dynamic changes of rescue vehicles, medical facility capacity, the dynamic changes of the survival probabilities of various types of wounded over time, and the changes of the wounded's psychological status, and constructed the post-earthquake wounded second level that maximizes the number of wounded survival and minimizes the psychological cost[8]. The dual-objective dynamic planning model of medical facility location selection and wounded transportation in the evacuation mode solves how to choose temporary hospitals and rear 
hospitals when medical resources are limited.

In recent years, domestic and foreign scholars have conducted extensive research on the problem models of location and route optimization problems, such as random demand[6], dynamic[2], and limited number of vehicles. However, domestic scholars often take the shortest total arrival time and the minimum total distribution cost as the optimization objectives in the research of post-earthquake material distribution center location and routing optimization[9]、[1]、[5], and some scholars consider the distribution time and cost as well as add the road safety of distribution route to the optimization model.

In summary, domestic and foreign scholars have relatively mature researches on the location of emergency facilities and routing optimization, but there are few studies on the location of rescue center facilities and routing optimization. This paper fully considers the urgency of rescue after the earthquake and the rescue needs of the wounded after the earthquake, and uses a two-stage heuristic algorithm to solve the location and route optimization of the emergency rescue center separately.First, minimize the cost of rescue center location as the first stage optimization goal, and use immune optimization algorithm to solve. Then, the shortest rescue path is taken as the second stage optimization goal, and simulated annealing algorithm and ant colony algorithm are used to solve them separately.

\section{PROBLEM DEFINITION FORMULATION}

AND

\subsection{Problem definition}

This article studies the post-earthquake emergency planning divided into two stages. The first stage is to select the location of the rescue center, and the second stage is to optimize the rescue route for the wounded. Regarding the location of the rescue center, when an earthquake occurs, the emergency operations center makes an overall plan based on the relevant disaster information. Given the location of $N$ disaster points and the number of wounded, $m_{\text {locations among the }}$ $N$ demand points are selected as rescue centers, and each disaster point is rescued by only one rescue center. On the premise of meeting the upper limit of the distance from the disaster point to the rescue center, the product of the travel distance between the rescue center and the disaster point and the rescue demand of the wounded is minimized.

\subsection{Formulation}

\subsubsection{Assumptions}

First of all, assumptions about the location of rescue centers. (1)A disaster site is served by only one rescue center; (2)The total rescue amount of each rescue center meets the demand of each disaster site rescued by it; (3) The location of each disaster site and the number of wounded are known. Then, the rescue path problem hypothesis. (1)The location of each disaster site and the number of wounded persons are known;(2)Each disaster site is rescued, and only once; (3)The total number of rescue vehicles rescued on each rescue path does not exceed its load capacity.

\subsubsection{Notations}

Symbol description of rescue center location problem: (1) $N$ indicates the set of disaster points; (2) $M_{i}$ indicates the set of candidate rescue centers whose distance to disaster poin $i$ is less than $d, i \in N, M_{i} \in N$; (3) $q_{i}$ represents the number of wounded at disaster point $i$; (4) ${ }^{d_{i j}}$ represents the distance from disaster point $i$ to the nearest rescue center $j$; (5) $m$ represents the number of rescue centers to be built; (6) $D$ represents the upper limit of the distance between the candidate rescue center and the disaster point it serves; (7) $y_{i j}=1$ means that disaster point $i \in N$ is rescued by rescue center $j \in M_{i}$, otherwise $y_{i i}=0$; (8) $x_{j}=1$ means that a rescue center is established in $j \in M_{i}$, otherwise $x_{j}=0$.Symbol description of rescue path optimization problem: 9) $N$ represents the collection of disaster points; (10) ${ }^{d i j}$ represents the distance between two points $i, j$; (11) $K$ represents the collection of rescue vehicles $k$;12 $q_{i}$ represents the number of wounded at the disaster site; (13) $Q$ represents the maximum load capacity of each rescue vehicle; (14) 0 represents the rescue center, $n+1$ represents the virtual rescue center;(15) ${ }^{x_{i j k}}=1$ means the rescue vehicle rescues from $i$ and $j$, otherwise $x_{i j k}=0$.

\subsubsection{Model description}

Site selection problem model formulation:

$$
\begin{aligned}
\min f_{1} & =\sum_{i \in N} \sum_{j \in M_{i}} q_{i} d_{i j} z_{i j} \\
\sum_{j \in M_{i}} z_{i j} & =1, i \in N
\end{aligned}
$$




$$
\begin{aligned}
& \sum_{j \in M_{i}} x_{j}=m \\
& d_{i j} \leq D \\
& z_{i j} \leq x_{j}, i \in N, j \in M_{i} \\
& x_{j} \in\{0,1\}, j \in M_{i} \\
& z_{i j} \in\{0,1\}, i \in N, j \in M_{i}
\end{aligned}
$$

The objective Equation (1)indicates that the sum of the product of the number of wounded and the distance between each rescue center and each disaster point is the smallest. Equation (2) means that each disaster spot is rescued by only one rescue center. Equation (3) means the number of rescue centers to be built. Equation (4) Disaster spot needs to be within the scope of rescue services that can be provided by the rescue center. Equation (5) means that only when a rescue center is built can rescue services be provided to disaster sites. Equation (6) and (7) indicate the value range of decision variables.

Route optimization problem model construction:

$$
\begin{aligned}
& \min f_{2}=\sum_{i=0}^{N} \sum_{j=0}^{N} \sum_{k=1}^{K} d_{i j} x_{i j k} \\
& \sum_{j=0}^{N} \sum_{k=0}^{K} x_{i j k}=1, \forall i \in N \\
& \sum_{j=0}^{N} x_{0 j k}=1, \forall k \in K \\
& \sum_{i=0}^{N} x_{i h k}-\sum_{j=0}^{N} x_{h j k}, \forall h \in N, k \in K \\
& \sum_{i=0}^{N} x_{i, n+1, k}=1, \forall k \in K \\
& \sum_{i=0}^{N} q_{i} x_{i j k} \leq Q, \forall k \in K \\
& x_{i j k} \in\{0,1\}, i, j \in N ; k \in K
\end{aligned}
$$

Equation (8) represents the shortest total rescue path.Equations (9) and (10) indicate that only one rescue vehicle provides rescue services at each disaster site. Equation (11) is the flow balance constraint of rescue vehicles on the path. Equation (12) indicates that each rescue vehicle needs to return to the rescue center. Equation (13) is the load capacity constraint of the rescue vehicle. Equation (14) indicates the value range of decision variables.

\section{ALGORITHM DESIGN}

\subsection{Immune Algorithm}

Applying the concept of immunity and its theory to genetic algorithms, while preserving the excellent characteristics of the original algorithm, try to selectively and purposefully use some characteristic information or knowledge in the problem to be sought to suppress the degradation phenomenon that occurs in the optimization process. This algorithm is called Immune Algorithm (Immune Algorithm) IA.

(1) Generation of initial antibody population. A simple coding method is used here. Each site selection plan can form an antibody of length $m$ ( $m$ represents the number of rescue centers), and each antibody represents a sequence of demand points selected as rescue centers.

(2) Evaluation of solution diversity.

(1)Affinity of antibody antigen. The affinity function is designed for the above-mentioned rescue center location model.

$$
A v=\frac{1}{F_{v}}=\frac{1}{\sum_{i \in N} \sum_{j \in M i} q_{i} d_{i j} z_{i j}-C \sum_{i \in N} \min \left\{\left[\sum_{j \in M_{i}} z_{i j}\right]-1,0\right\}}
$$

Among them, $F_{v}$ represents the new objective function, the second term of the denominator represents penalties for solutions that violate the distance constraint, and $C$ takes a larger positive number.

(2)Affinity between antibody and antibody.

$S_{v, s}=\frac{k_{v, s}}{L}$

Among them, ${ }^{k_{v, s}}$ represents the same number of bits between antibody $v$ and antibody $\boldsymbol{S}$, and $L$ is the total length of the antibody.

(3) Antibody concentration. The antibody concentration $C_{v}$ is the proportion of similar antibodies in the population.

$C_{v}=\frac{1}{N} \sum_{j \in N} S_{v, s}$

$S_{v, s}=\left\{\begin{array}{l}1, S_{v, s}>T \\ 0, \text { 其他 }\end{array}\right.$

$N$ represents the total number of antibodies, and $T$ is the preset threshold.

(4) Expected reproduction probability.

The calculation formula is:

$$
P=\alpha \underset{\sum_{\text {is a constant. }}}{\sum A_{v}}+(1-\alpha) \frac{C_{v}}{\sum C_{v}}
$$

(3) Immune operation. Selection: According to the roulette mechanism, the probability of an individual being selected is the expected reproduction probability. Crossover: Use single point crossover method for crossover operation. Mutation: Use random mutation bits for mutation operation.

\subsection{Ant colony algorithm}

When ants look for food, they release pheromones along the path they travel, and they can sense the pheromones released by other ants. The concentration of pheromone is used to characterize the distance of the 
path, the higher the concentration, the shorter the path.

(1)Initialization parameters. At the beginning of calculation, relevant parameters need to be initialized.

(2)Construct solution space.

$$
\eta_{i j}(t)=\frac{1}{d_{i j}} \text { represents }
$$

the degree of expectation of ants from $i$ to $j$. allow $(k)$ represents the collection of ants to visit disaster points.

$P_{i j}^{k}=\left\{\begin{array}{l}\frac{\left[\tau_{i j}(t)\right]^{\alpha}\left[\eta_{i j}(t)\right]^{\beta}}{\sum_{j \in \text { allow }(k)}\left[\tau_{i j}(t)\right]^{\alpha}\left[\eta_{i j}(t)\right]^{\beta}}, j \in \operatorname{allow}(k) \\ 0, j \notin \text { allow }(k)\end{array}\right.$

(3) Update pheromone.

Pheromone release formula:

$\tau_{i j}(\mathrm{t})=\frac{Q}{L_{k}}$

$L_{k}$ represents the length of the path passed by the $k$ th ant.

Pheromone update formula:

$\tau_{i j}(t+1)=(1-\rho) \tau_{i j}(t)+\tau_{i j}$

$\tau_{i j}=\sum_{k=1}^{m} \tau_{i j}^{k}$

Among them, $\tau_{i j}^{k}$ represents the pheromone concentration released by the $k$ th ant on the path connecting disaster point $i$ and disaster point $j$, and $\tau_{i j}$ represents the sum of the pheromone concentration released by all ants on the path connecting disaster point $i$ and disaster point $j$.

(4)Determine whether to terminate. If iter $<$ iter $-\max$, let iter $=$ iter +1 , clear the record table of the ant's path and return to step (2), otherwise, terminate the calculation and output the optimal solution.

\subsection{Simulated annealing algorithm}

The idea of Simulated Annealing (SA) was first proposed by Metropolis et al. The starting point is based on the similarity between the annealing process of solid substances in physics and the general combinatorial optimization problem. Simulated annealing is a general optimization algorithm

After a given domain structure, the simulated annealing process is a continuous random walk from one state $i$ to another state $j$. When the temperature $T$ is a certain value, the probability from state ${ }_{i}$ to state $j$ is:

$P(T)=\left\{\begin{array}{l}1, E(i) \geq E(j) \\ \exp \left(-\frac{E(j)-E(i)}{T}\right), E(i)<E(j)\end{array}\right.$

(1)Initialization parameters. Set the initial temperature $T_{0}$, the end temperature $T_{k}$, and randomly generate the initial solution, which is used as the current best point to calculate the objective function.

(2) Generate new solutions. Randomly disturb the current best point to generate new solutions.

(3) Calculate the difference between the new and old solutions. $E(s)=E(j)-E(i)$

(4) Determine whether to accept the new solution. If $E(s) \leq 0$, accept the new solution, if $E(s)>0$, when the probability $P$ is greater than a random number between 0 and 1, then accept the new solution;

(5) Determine whether the number of cycles is reached. When the number of iterations of the inner loop is reached, it jumps out of the inner loop, cools down to $T_{0} \leq T_{k}$, and terminates the outer loop iteration.

\section{NUMERICAL ANALYSIS}

\subsection{Source of data}

The data in this article refers to [4], using a total of 30 disaster site.The solution results and analysis of rescue site selection and route optimization problems. Use MATLAB2019b software to solve the optimization problem, the algorithms involved are immune optimization algorithm, ant colony algorithm and simulated annealing algorithm. The calculation results are as follows:

\subsection{Solution results and analysis}

Table 1.Results of solving the problem of rescue center location

\begin{tabular}{c|c|l|c|} 
Solution & Rescue center number & 1 (The disaster site number is 16) & 2 (The disaster site number is 23) \\
\hline $\begin{array}{c}\text { Immune } \\
\text { optimization }\end{array}$ & Disaster site number & $3,4,5,6,7,8,9,10,14,15$ & $1,2,11,12,13,17,18,19,20,21,22,24,25,26$ \\
& & & $, 27,28,29,30$ \\
\hline
\end{tabular}


Table 2.Route optimization problem solving result

\begin{tabular}{|c|c|c|c|c|}
\hline Solution & $\begin{array}{l}\text { Rescue } \\
\text { center }\end{array}$ & Rescue route & $\begin{array}{l}\text { Number of } \\
\text { vehicles }\end{array}$ & $\begin{array}{c}\text { Objective } \\
\text { function value }\end{array}$ \\
\hline \multirow{8}{*}{$\begin{array}{l}\text { Ant colony } \\
\text { algorithm }\end{array}$} & \multirow[t]{3}{*}{1} & center $1 \rightarrow 6 \rightarrow 5 \rightarrow 7 \rightarrow$ Rescue center 1 & \multirow[t]{8}{*}{8} & \multirow[t]{8}{*}{36.3766} \\
\hline & & Rescue center $1 \rightarrow 15 \rightarrow 14 \rightarrow 3 \rightarrow 4 \rightarrow$ Rescue center 1 & & \\
\hline & & Rescue center $1 \rightarrow 8 \rightarrow 10 \rightarrow 9 \rightarrow$ Rescue center 1 & & \\
\hline & \multirow[t]{5}{*}{2} & Rescue center $2 \rightarrow 22 \rightarrow 13 \rightarrow 12 \rightarrow 11 \rightarrow$ Rescue center 2 & & \\
\hline & & Rescue center $2 \rightarrow 24 \rightarrow 20 \rightarrow 19 \rightarrow$ Rescue center 2 & & \\
\hline & & Rescue center $2 \rightarrow 27 \rightarrow 26 \rightarrow 21 \rightarrow 17 \rightarrow 18 \rightarrow$ Rescue center 2 & & \\
\hline & & Rescue center $2 \rightarrow 28 \rightarrow 8 \rightarrow 30 \rightarrow$ Rescue center 2 & & \\
\hline & & Rescue center $2 \rightarrow 25 \rightarrow 2 \rightarrow 1 \rightarrow$ Rescue center 2 & & \\
\hline \multirow{6}{*}{$\begin{array}{l}\text { Simulated } \\
\text { annealing } \\
\text { algorithm }\end{array}$} & \multirow[t]{2}{*}{1} & Rescue center $1 \rightarrow 7 \rightarrow 6 \rightarrow 4 \rightarrow 3 \rightarrow 5 \rightarrow$ Rescue center 1 & \multirow[t]{6}{*}{6} & \multirow[t]{6}{*}{31.7106} \\
\hline & & Rescue center $1 \rightarrow 8 \rightarrow 9 \rightarrow 10 \rightarrow 14 \rightarrow 15 \rightarrow$ Rescue center 1 & & \\
\hline & \multirow[t]{4}{*}{2} & Rescue center $2 \rightarrow 25 \rightarrow 2 \rightarrow 1 \rightarrow 30 \rightarrow$ Rescue center 2 & & \\
\hline & & $\begin{array}{c}\text { Rescue center } 2 \rightarrow 17 \rightarrow 21 \rightarrow 26 \rightarrow 28 \rightarrow 29 \rightarrow 27 \rightarrow \text { Rescue center } \\
2\end{array}$ & & \\
\hline & & Rescue center $2 \rightarrow 19 \rightarrow 20 \rightarrow 24 \rightarrow$ Rescue center 2 & & \\
\hline & & Rescue center $2 \rightarrow 22 \rightarrow 18 \rightarrow 12 \rightarrow 11 \rightarrow 13 \rightarrow$ Rescue center 2 & & \\
\hline
\end{tabular}

Comparing the solution results of the ant colony algorithm and the simulated annealing algorithm, it can be seen that the rescue path obtained by the simulated annealing algorithm is shorter and the number of rescue vehicles used is less, which is conducive to saving rescue resources and saving rescue costs.

\section{CONCLUSION}

In this paper, a two-stage solution model of location and route is established according to the problem of post-earthquake wounded rescue. Firstly, minimizing the location cost of rescue center is taken as the optimization goal of the first stage, and immune optimization algorithm is used to solve the problem, and a reasonable location of rescue center is selected. Then, according to the location of the rescue center selected in the first stage, a two-stage mathematical programming model for the rescue of the wounded after the earthquake is established, and simulated annealing algorithm and ant colony algorithm are designed to solve the problem, and the results of these two algorithms are compared and analyzed.

The solution results show that the rescue path obtained by the simulated annealing algorithm is shorter, and the number of rescue vehicles used is less, which is beneficial to saving rescue resources and saving rescue costs.
This article only studies the location and route optimization for the rescue of the injured after the earthquake, without taking into account the subsequent transportation of the injured. Therefore, how to rescue the injured and the transportation in an uncertain environment is also a future research direction.

\section{AUTHORS' CONTRIBUTIONS}

In this paper, a two-stage mathematical programming model is established to study the rescue of the wounded after the earthquake. First, immune optimization algorithm is used to select the rescue center from many disaster points, and then the path planning model of the wounded rescue vehicle with the shortest total rescue path is established, and simulated annealing algorithm and ant colony algorithm are designed to solve it. The results show that the rescue path obtained by simulated annealing algorithm is shorter, and the number of rescue vehicles used is less, thus saving the rescue cost. It is helpful for emergency decision-makers to determine the reasonable number of rescue vehicles.

\section{REFERENCES}

[1] Chang Shi. Research on model and algorithm of location-routing (LRP) for emergency logistics system in post-earthquake[D]. Chengdu: University 
of Electronic Science and Technology of China, 2016.

[2] Changshi Liu, Gang Kou, Daobo Liu. Fuzzy dynamic LRP for post-earthquake multimodal relief delivery [J]. Journal of management sciences in CHINA, 2016, 19(10): 61-72.

[3] Firat, K., B. Y. Kara, and B. Bozkaya. Locating temporary shelter areas after an earthquake: A case for Turkey[J]. European Journal of Operational Research,2015,243(1).

[4] Fuyu Wang, Cunming Ye, Tao Wang and Jingjing Zhao. Research on two stage planning model and algorithm of wounded rescue vehicle after earthquake $[\mathrm{J}]$. Journal of management sciences in CHINA, 2018, 21(02): 68-79.

[5] Haijun Wang, Lijing Du and Shihua Ma. Model and algorithms for integrated open location and routing problem in emergency logistics under earthquake $[\mathrm{J}]$. Journal of industrial engineering/engineering management, 2016,30(02):108-115.
[6] Huiling Zhong, Nan Zhuang, Guanxiang Zhang and Wenxue Cai. $\alpha$-robust emergency facilities location problems for hazardous materials transportion[J]. Systems engineering-theory \& practice, 2013,33(05):1262-1268.(in Chinese)

[7] Jomon A. P., L. MacDonald. Location and capacity allocations decisions to mitigate the impacts of unexpected disasters $[\mathrm{J}]$. European Journal of Operational Research, 2016, 251(1): 252-263.

[8] Huali Sun, Liping Cai, Ling Zhang and Zhe Zhao. Multi-objective dynamic facility location-transportion problem in emergency humanitarian logistics[J]. Chinese journal of management science, 2020,28(03):103-112.

[9] Shuanglin Li, Zujun Ma, Bin Zheng and Ying Dai. Fuzzy mulit-objective location-multimodal transportation problem for relief delivery during the initial post-earthquake period[J]. Chinese journal of management science, 2013,21(02):144-151. 